\title{
A data-driven approach for fast controller calibration of Brake-by-Wire actuators
}

\author{
Stefano Radrizzani, Davide Todeschini, Giorgio Riva, \\ Simone Formentin, Giulio Panzani and Sergio M. Savaresi
}

\begin{abstract}
Brake-by-Wire (BBW) actuators are the current frontier of the Ride-by-Wire paradigm applied to modern and future vehicles. The control of such actuators has reached a certain scientific maturity, but the calibration of the proposed solutions is strongly dependent on the availability of a system model. In the pursuit of a faster, automatic and possibly continuous - during the component lifetime - calibration of the control logic, data-driven model-free tuning techniques represent an interesting opportunity. In this paper, this topic is addressed by using the Virtual Reference Feedback Tuning (VRFT) approach, applied on the BBW cascade control architecture in [1]. The data-driven tuning shows equivalent closed-loop performances with respect to the standard modelbased approach, adding two benefits: the simpler (thus faster) experimental campaign to collect the necessary data and the possibility of using normal system operation data for the tuning, paving the way for the automatic maintenance of such systems, which suffers the effect of ageing and wearing.
\end{abstract}

\section{INTRODUCTION}

The application of the so-called Ride-by-Wire paradigm, where the driver commands are physically decoupled from the vehicle and are interpreted by an electronic control unit, eventually commanding independent actuators, has certainly supported the increased level of performance reached by vehicle dynamics control systems. Such paradigm has been firstly applied to the throttle command (Throttle-by-Wire, TBW) and lately to the vehicle brakes with the so-called Brake-by-Wire (BBW) actuators. The Ride-by-Wire trend is also supported by the strong interest in the autonomous driving, which requires the availability of extremely precise and, needless to say, autonomous actuators. It is also worth noticing that the diffusion of hybrid and electric vehicles pushes in the same direction, making possible a more sophisticated and safe management of the trade-off between traditional and regenerative braking: when electric motors cannot absorb a sufficient level of energy to provide a performing braking manoeuvrer, traditional brakes are electronically activated to actuate an additional braking torque [2].

In this work, the braking pressure control of a hydraulic BBW actuator is addressed. This topic is not new in literature, in particular its application in racing contexts [3], [1], [4] that require development of advanced control strategies. Some of them deal with the design of a robust friction compensation [5] or an adaptive gap covering when the pads are not in contact with the disk [6]. All the discussed solutions are grounded on model-based control strategies, therefore

\footnotetext{
*All the authors are with the Department of Electronics, Information and Bioengineering, Politecnico di Milano, 20133 Milan, Italy giulio.panzaniepolimi.it
}

suffer of two main drawbacks: the first one is the non trivial automatization of the controller tuning procedure and the second is that extensive and ad-hoc experimental campaigns are usually required for the proper system modelling.

On the other side, these efforts have led to a deep understanding of the BBW systems and to a solid scientific maturity in their control: this opens the way to exploring the employment of data-driven model-free techniques for their automatic calibration. Within this perspective, in this paper the focus is set on the development and experimental verification of a data-driven complete calibration of a previously designed model-based control system [1], that features a two loops cascade architecture and a feed-forward action. To achieve this goal, the Virtual Reference Feedback Tuning (VRFT) [7] for cascade control systems [8], [9] is employed, while the feed-forward action, characterized by a staticmap, is reshaped by solving a curve fitting problem. None of the employed techniques require a model of the plant, thus making the recalibration procedure robust to system variations and modelling errors.

The reminder of the paper is organized as follows. Section II presents an overview of the system and the experimental setup; in Section III a model-based control is designed, providing the benchmark state of the art method. In Section IV the proposed data-driven calibration is discussed and experimental results are shown to assess the proposed approach and compare it with the model-based. The paper ends with some concluding remarks.

\section{SYSTEM OVERVIEW}

A Brake-by-Wire (BBW) actuator is a braking system where the pressure generated on the disks by the pads is regulated by an electronic control unit (ECU) and not directly by the driver. The BBW involved in this work is sketched in Fig. 1, where the main subsystems are highlighted: the electric part (DC brushless motor and power converter), the mechanical one (gear wheels and screw-and-nut) and the hydraulic one (master cylinder, caliper and pipeline). The actuator is equipped with a pressure sensor in the master cylinder and an encoder, which provides the position of the piston. A low level controller (with a bandwidth of $5 \mathrm{kHz}$ ) allows to regulate the motor current, eventually responsible for the piston movement and braking pressure generation. All the experiments have been performed on a static test bench where the disks are not rotating and in contact with the pads; the controllers and the data $\log$ run with a task frequency of $500 \mathrm{~Hz}$. 


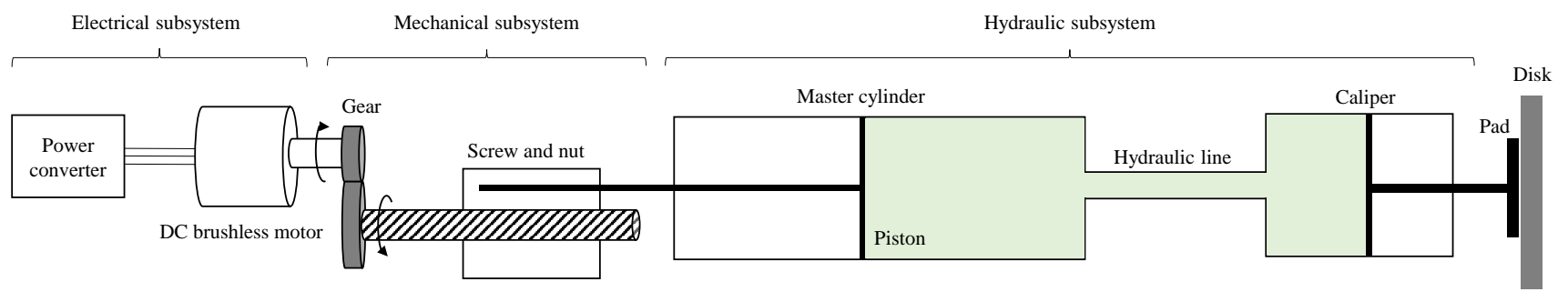

Fig. 1. The BBW actuator sketch.

\section{A. Modelling}

The physical model of a BBW actuator with the same layout has been previously discussed in different works [3], [4]. Due to the significant length of the hydraulic line of the system, the discussed models are extended so to evaluate its effect. In particular, the proposed state-space model is given by the nonlinear set of equations (1):

$$
\begin{array}{r}
\dot{x}_{M C}=K_{G} \Omega \\
\dot{\Omega}=\frac{\eta_{G} K_{M} I-K_{G}\left(p A_{M C}+c_{D} K_{G} \Omega+F_{F R}\right)}{\eta_{G} J_{M}+K_{G}^{2} m} \\
\dot{p}=\frac{\beta_{e f f}}{A_{M C}\left(s_{M C}-x_{M C}\right)}\left(-q_{M C}+A_{M C} K_{G} \Omega\right) \\
\dot{p}_{P D}=\frac{K_{P D}}{A_{P D}^{2}} q_{P D} \\
\dot{p}_{P L}=\frac{\beta_{e f f}}{A_{P L} L_{P L}}\left(q_{M C}-q_{P D}\right) \\
\dot{q}_{M C}=\frac{A_{P L}}{\rho L_{P L}}\left(2\left(p-p_{P L}\right)-K_{P L} q_{M C}-\rho g \Delta h\right) \\
\dot{q}_{P D}=\frac{A_{P L}}{\rho L_{P L}}\left(2\left(p_{P L}-p_{P D}\right)-K_{P L} q_{P D}-\rho g \Delta h\right)
\end{array}
$$

where (1b) describes the mechanical power balance, (1c) and (1d) are force balances in the master cylinder and the caliper, assuming an elastic contact between pads and disk. Finally, the last three equations represent the pressure dynamics with its propagation through the pipeline. This model is derived assuming constant density and viscosity of the oil, constant Bulk modulus $\beta$ whose equivalent effective value takes into account the possible presence of air in the hydraulic circuit in case of non-perfect bleeding. The complete list of model parameters and signals is provided in Table I.

Fig. 2 shows the bode diagrams of the the transfer functions of the linearised system between the current and the two outputs, using different values of pipeline length in order to highlight its role in the system dynamics. The pipeline effect $\left(L_{P L} \neq 0\right)$ is particularly significant in the current-pressure transfer function, causing second-order underdamped resonance peaks in the diagram, while it is negligible in the current-position one. Moreover, the longer the pipeline, the lower are the frequencies which are affected by its presence.

\section{B. Control architecture}

Considering the presence of the pressure dynamics the design of a direct pressure controller results complicated,
TABLE I

PARAMETERS AND SIGNALS

\begin{tabular}{l|c||l|c}
\hline \multicolumn{2}{c||}{ Parameters } & pipeline length & $L_{P L}$ \\
\hline gear ratio & $K_{G}$ & pipeline resistance & $K_{P L}$ \\
\hline gear efficiency & $\eta_{G}$ & pipeline inlet-outlet height & $\Delta h$ \\
\hline motor constant & $K_{M}$ & gravity acceleration & $g$ \\
\hline motor inertia & $J_{M}$ & \multicolumn{2}{c}{ Signals } \\
\hline damping coefficient & $c_{D}$ & piston position & $x$ \\
\hline oil mass & $m$ & motor speed & $\Omega$ \\
\hline oil density & $\rho$ & motor current & $I$ \\
\hline effective Bulk modulus & $\beta_{e f f}$ & friction force & $F_{F R}$ \\
\hline master cylinder section & $A_{M C}$ & master cylinder pressure & $p$ \\
\hline piston stroke & $s_{M C}$ & pads pressure & $p_{P D}$ \\
\hline load elasticity & $K_{P D}$ & pipeline pressure & $p_{P L}$ \\
\hline pads contact area & $A_{P D}$ & pipeline inlet flowrate & $q_{M C}$ \\
\hline pipeline section & $A_{P L}$ & pipeline outlet flowrate & $q_{P D}$ \\
\hline
\end{tabular}
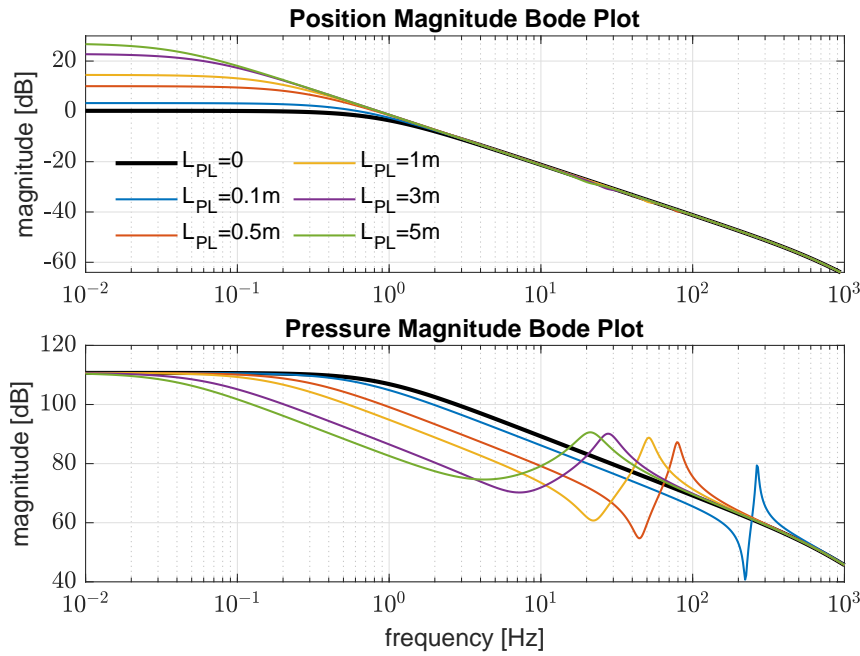

Fig. 2. Sensitivity of the linearized model Bode magnitude plots with respect to the pipeline length $L_{P L}$.

even due to the variability of the dynamics with respect to system conditions, such as temperature and bleeding status. Therefore the cascade control architecture proposed in [1] suits well for the BBW control. A high bandwidth internal position loop is firstly designed, then the pressure control is achieved by an external loop. Moreover, a position feedforward action is computed from the pressure reference, so to enhance tracking performance and to account for the static system nonlinearities between the position and the braking pressure - summarized in the static position-pressure map in Fig. 3 - due to a non perfect linear elastic contact between the 
disk and the pads. A schematic representation of the control architecture is reported in Fig. 4. In order to mitigate the

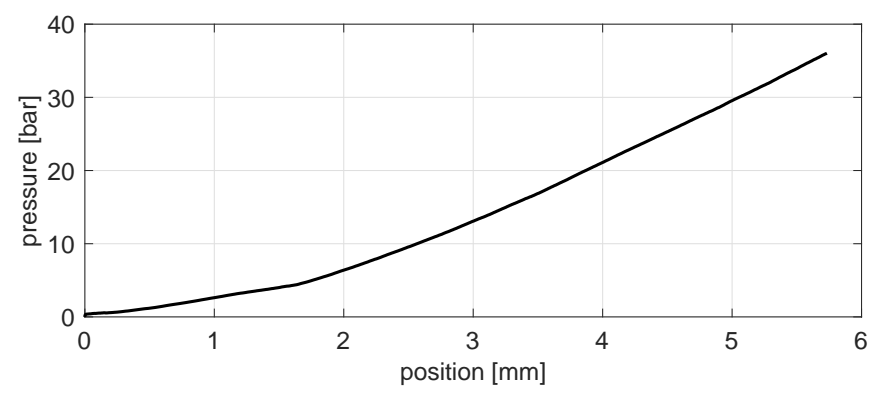

Fig. 3. Nonlinear position-pressure map typical of BBW actuators.

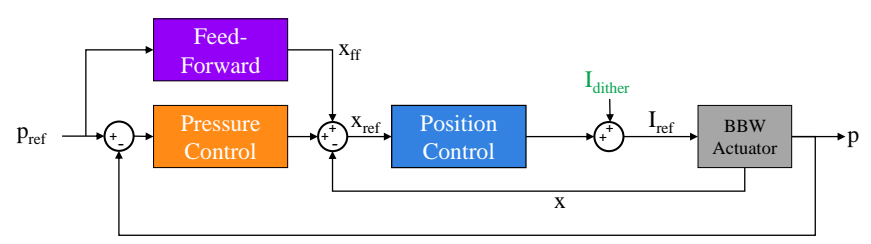

Fig. 4. Control architecture.

effects of the friction $F_{F R}$ that appears in (1b), a current dither [5] is also added to the control input. Its frequency is set constant to $50 \mathrm{~Hz}$, outside the target bandwidth of the controlled system, and its amplitude increases linearly with the pressure.

\section{MODEL-BASED CONTROL DESIGN}

In order to tune all the parameters of the control architecture shown in Fig. 4, using a traditional model-based design paradigm, a significant number of steps is necessary:

A. Current-position dynamics identification (int. loop);

$B$. Position control tuning and validation (int. loop);

C. Position-pressure dynamics identification (ext. loop);

$D$. Pressure control tuning and validation (ext. loop);

$E$. Feed-forward design and calibration (feed-forward).

\section{A. Current-position dynamics identification}

The first step is the identification of the current-position transfer function. Data are collected using the PRBS (Pseudo-Random Binary Sequence) as current input. Bias, amplitude and switching time are chosen to be in a working point such that the current is sufficiently low to collect a long amount of data without stressing the power converter, but sufficiently high to consider the system in pressure conditions. The estimated Bode plot of the transfer function is in Fig. 5 and it is coherent with the model analysis; in fact the current-position dynamics is well described by a damped second order linear system, featuring complex poles at 9.1 $\mathrm{Hz}$ and 0.77 as damping ratio.

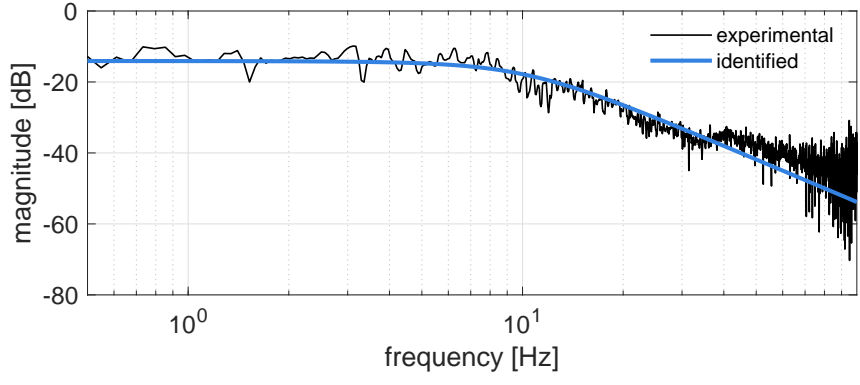

Fig. 5. Magnitude Bode plot of the current-position dynamics estimated on experimental data: the identified current-position transfer function is highlighted.

\section{B. Position control tuning and validation}

A classical model-based control technique is the loopshaping [10]. Given the identified second order dynamics, a PID control can be used to close the position loop. Its zeros are placed in correspondence of the identified poles, while the gain is increased so to yield a bandwidth of $40 \mathrm{~Hz}$. The resulting control parameters for the position (internal) loop are listed in Table II.

\section{Position-pressure dynamics identification}

In order to design the pressure loop, the dynamics between the position reference and the pressure are then identified. Similarly to the internal dynamics identification, a PRBS signal is used as position setpoint. Fig. 6 shows how relevant the pressure resonance peak is, while a constant gain represents well the lower frequencies.

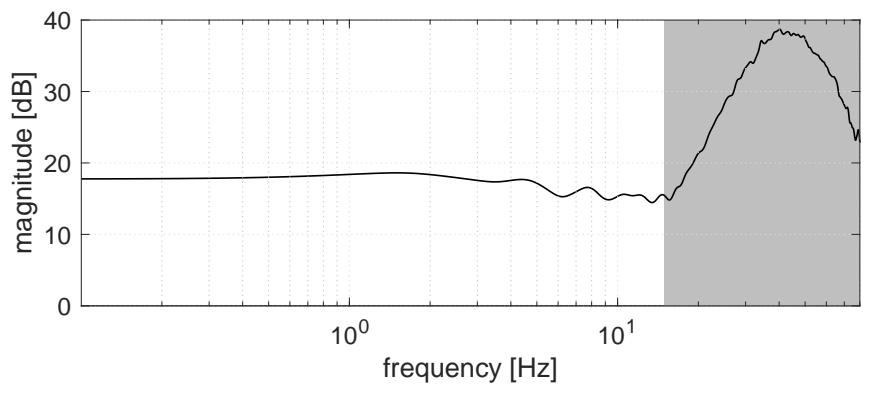

Fig. 6. Magnitude Bode plot of the position-pressure dynamics estimated on experimental data, once the position loop is closed. The frequency region affected by pipeline dynamics highlighted.

\section{Pressure control tuning and validation}

The pressure control is closed at a sufficiently low bandwidth, so to consider the pressure dynamics negligible. Therefore, a PI control can be designed, whose gain is set such that the closed-loop reaches a bandwidth of $1 \mathrm{~Hz}$, while the zero can be used to increase the phase margin. The final control parameters for the pressure (external) loop are listed in Table II, while in Fig. 10 a time-domain pressure step response is shown. 


\section{E. Feed-forward design}

The last block to be designed is the feed-forward action, that is characterized by the nonlinear map in Fig. 7, that consists in an inverted branch of parabola fitted on the steady-state position-pressure data, collected performing a slow position ramp up to a significant pressure level. In order

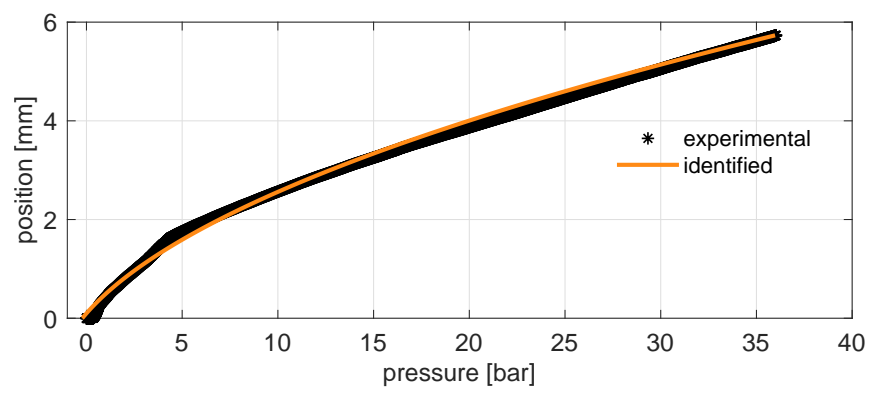

Fig. 7. Nonlinear feed-forward map fitted on experimental data.

to appreciate the importance of the feed-forward contribution, Fig. 8 shows the tracking of a real braking profile with and without the inclusion of the feed-forward term.

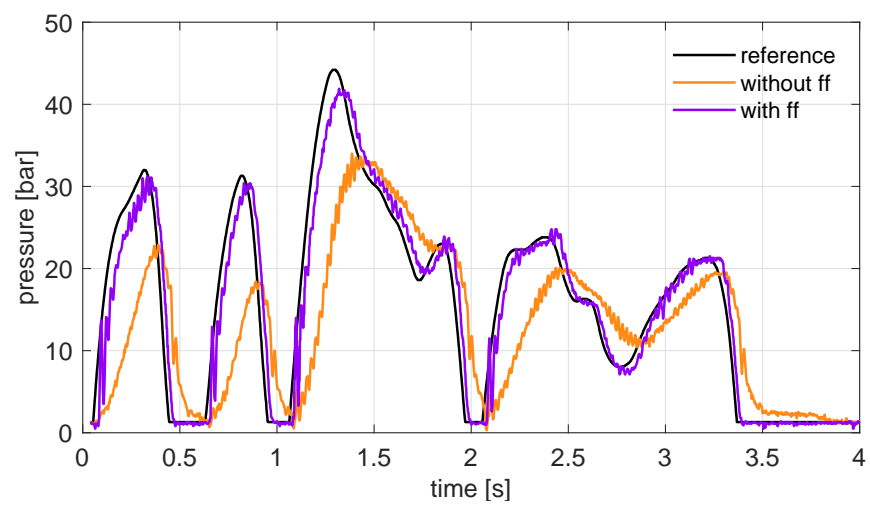

Fig. 8. Real braking profile response of the pressure controlled system with and without feed-forward (ff).

\section{DATA-DRIVEN CONTROL RECALIBRATION}

For the model-based controller described in Section III a significant loss of performance may occur if the system changes from the identified one; this might actually happen during the BBW lifetime because of mechanical wearing, ageing or actuator and pad renewal. This situation may be overcome with a complete re-tuning of the controller but the model-based technique is time consuming, as it requires several experiments and design steps. In order to address a faster and simpler controller recalibration, a data-driven

TABLE II

MODEL-BASED CONTROL PARAMETERS

\begin{tabular}{l|c|c|c|c}
\hline & \multicolumn{2}{|c|}{ Position Control } & \multicolumn{2}{c}{ Pressure Control } \\
\hline proportional gain & $\frac{A}{m m}$ & 14.6244 & $\frac{m m}{b a r}$ & 0.0032 \\
\hline integral gain & $\frac{A}{m m \cdot s}$ & 494.4472 & $\frac{m m}{b a r} \cdot s$ & 0.8055 \\
\hline derivative gain & $\frac{A \cdot s}{\mathrm{~mm}}$ & 0.1684 & - & - \\
\hline
\end{tabular}

control tuning is here presented, which eventually will be shown to be suitable for a recursive and automatic execution during the whole component lifetime.

The proposed data-driven control recalibration is characterized by two steps: one where the feed-forward map is identified, and the other to tune both feedback controller parameters. The first goal is achieved by means of a curve fitting problem, while the second one thanks to the Virtual Reference Feedback Tuning (VRFT) for cascade control systems.

\section{A. Feed-forward automatic recalibration}

The feed-forward map learning is formulated as a curve fitting problem. In fact, it has been shown that the positionpressure map is well approximated by a parabola, therefore the problem can be formulated as in (2):

$$
\left\{\begin{array}{l}
\min _{\theta} J(t)=\min _{\theta} \frac{1}{t} \sum_{k=1}^{t} \mu^{t+1-k}\left(p(k)-\phi^{T}(k) \theta\right)^{2} \\
\phi^{T}(t) \theta=\left[\begin{array}{lll}
x^{2}(t) x(t) & 1
\end{array}\right]\left[\begin{array}{l}
a \\
b \\
c
\end{array}\right]
\end{array}\right.
$$

where the parameter $\mu$ plays the role of a forgetting factor, by weighting the past values and $\theta^{T}=[a b c]$ is the vector of the parabola coefficients. It is clear that this formulation satisfies the property to be linear in the parameters, therefore the problem can be solved with Recursive Least-Square (RLS) algorithm [11], that is characterized by the set of equations in (3):

$$
\left\{\begin{array}{l}
\theta(t)=\theta(t-1)+\frac{V(t-1) \phi(t)}{1+r(t)}\left(p(t)-\phi^{T}(t) \theta(t-1)\right) \\
V(t)=\frac{1}{\mu}\left[V(t-1)-\frac{V(t-1) \phi(t) \phi(t)^{T} V(t-1)}{1+r(t)}\right] \\
r(t)=1+\phi(t)^{T} V(t-1) \phi(t)
\end{array}\right.
$$

where $\mu, \theta(0)$ and $V(0)$ are design parameters. For this application, they are chosen as follows: $\mu$ fixed to one because the quantity of data is limited in time, so all data are important; $\theta(0)$ is an initial guess of the map, based on previous experience; $V(0)$ is set equal to the identity matrix. Once learned the map, the right-branch of the parabola must be inverted to be used as feed-forward in the control action:

$$
x_{f f}=x^{0}+\sqrt{\frac{p_{r e f}-p^{0}}{a}}
$$

where $\left(x^{0}, p^{0}\right)$ are the coordinates of the minimum of the parabola. The choice to use a two-steps solution and not directly identify the curve expression in (4) is supported by the possibility of using an algorithm for linear problems.

Given its recursive formulation, this method can be used with ad-hoc experiments like the slow ramp used in the model-based approach, or it can even be used during the realtime operations as shown in Fig. 9; as expected the initial wrong guess of the map is progressively corrected by the new data that, after a transient, correctly converges to the final value. The final parabola well matches the experimental data, as it is visible from the upper plot of Fig. 9. 

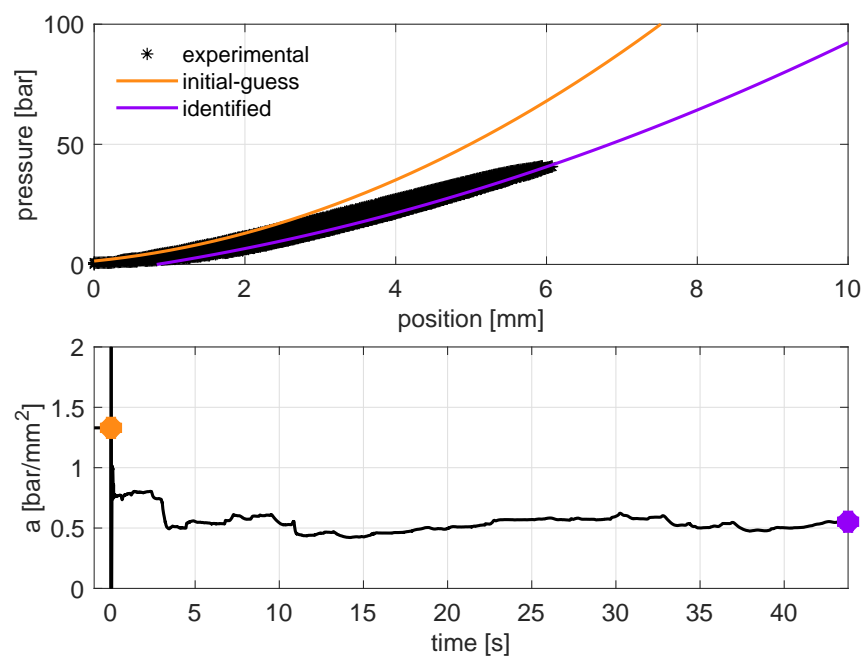

Fig. 9. Map learned from experimental data, given an initial wrong guess (top) and time evolution of parameter $a$ (bottom).

\section{B. Feedback control recalibration via VRFT}

VRFT is an offline direct data-driven method, usually employed for the design of parametric controllers without any previous system knowledge [7]. In this application, it is used to recalibrate an existing model-based controller, therefore the knowledge of the system will be used in order to tune properly the design-parameters of the VRFT approach. In particular, VRFT for cascade systems [8] consists in the solution of two optimization problems, one for each loop, starting from a single open-loop experiment $(\tilde{I}, \tilde{x}, \tilde{p})$. In the present context, the employed experiment is the same PRBS one used for the identification of the current-position dynamics as in Section III-A.

The first problem to be solved is the internal loop one, equivalent to the traditional VRFT [7], whose aim is the minimization of the mismatch in (5) between a closed-loop reference model $M_{i}(s)$ and the actual closed-loop system weighted over the range of frequencies $W_{i}(s)$ :

$$
\min _{\theta_{i}} J_{M R}\left(\theta_{i}\right)=\min _{\theta_{i}}\left\|M_{i}(s)-\frac{C_{i}\left(\theta_{i}, s\right) G(s)}{1+C_{i}\left(\theta_{i}, s\right) G(s)}\right\|_{2}
$$

where $G(s)$ is the system transfer function and $\mathscr{C}$ is the class of parametric PID in (6).

$$
\begin{gathered}
\mathscr{C}=\left\{C_{i}\left(s, \theta_{i}\right) \mid C_{i}\left(s, \theta_{i}\right)=\beta_{i}^{T}(s) \theta_{i},\right. \\
\left.\beta_{i}(s)=\left[1 ; \frac{1}{s} ; \frac{s}{\frac{s}{2 \pi \cdot 100}+1}\right] \theta_{i} \in \mathbb{R}^{3}\right\}
\end{gathered}
$$

Noticing that $\exists C_{i}\left(\theta_{o p t}, s\right) \in \mathscr{C}: M_{i}(s)=\frac{C_{i}\left(\theta_{o p t}, s\right) G(s)}{1+C_{i}\left(\theta_{o} p t, s\right) G(s)} \Rightarrow$ $\tilde{I}=C_{i}\left(\theta_{\text {opt }}, s\right)\left(1-M^{-1}(s)\right)$, the cost function in (5) can be rewritten in a data-oriented way:

$$
\min _{\theta_{i}} J_{V R}^{N}\left(\theta_{i}\right)=\min _{\theta_{i}} \frac{1}{N} \sum_{k=1}^{N}\left(\left(\tilde{I}(k)-\gamma^{T}(k) \theta_{i}\right)\right)^{2}
$$

where $e_{i}(t)=\left(1-M_{i}^{-1}(s)\right) \tilde{x}(t)$ is the virtual error appearing in $\gamma^{T}(t)=\beta_{i}^{T}(s) e_{i}(t)$. Then all signals are filtered by the optimal filter $L_{i}(s)=M_{i}(s)\left(1-M_{i}(s)\right) \frac{W_{i}(s)}{U_{i}(s)}$ [7] that makes converge the minimum of (7) to the same one of (5), when the number of samples $N$ tends to infinity. Finally, the parameter $U_{i}(s)$ contains the information of the spectrum $\Phi_{I}(\omega)$ of the input, in fact $\left|U_{i}(j \omega)\right|^{2}=\Phi_{I}(\omega)$. When the controller is in the class of controller linear in the parameters, the solution can be achieved using Least-Squares (LS):

$$
\theta_{i}^{*}=\left[\sum_{t=1}^{N} \gamma_{L}(t) \gamma_{L}^{T}(t)\right]^{-1} \sum_{t=1}^{N}\left(\gamma_{L}(t) \tilde{I}_{L}(t)\right)
$$

where the subscript $L$ means that a signal is filtered by $L_{i}(s)$.

The challenging part of the VRFT is the choice of the reference model and the frequency weight. A second order reference model represents well the internal loop. Anyway, the system is subject to different disturbances, such as the dither and the static friction that make the matching with the model-reference over the complete range of frequency impossible. To reduce their effects on data, the designed frequency weight is very pendent after the cut-off frequency. The design parameters are in Table III.

Once designed a minimum-phase internal controller, VRFT for cascade system creates the virtual input to be used for the tuning of the external:

$$
\tilde{x}_{r e f}(t)=C_{i}^{-1}\left(s, \theta_{i}^{*}\right) \tilde{I}(t)+\tilde{x}(t)
$$

Then, the same steps of the internal one have to be followed. Given that a $1 \mathrm{~Hz}$ bandwidth is the goal, a simple first order system is used both as reference model and as frequency weight. Their parameters are in Table III.

TABLE III

VRFT DESIGN PARAMETERS

\begin{tabular}{c|c}
\hline Position Control & Pressure Control \\
\hline$M_{i}(s)=\frac{1}{\left(\frac{s}{2 \pi f_{i}}\right)^{2}+2 \cdot 0.85 \frac{s}{2 \pi f_{i}}+1}$ & $M_{o}(s)=\frac{1}{\frac{s}{2 \pi}+1}$ \\
\hline$W_{i}(s)=\frac{1}{\left(\frac{s}{1 \cdot 2 \cdot 2 \pi f_{i}}+1\right)^{7}}$ & $W_{o}(s)=\frac{W_{i}(s)}{\frac{s}{1 \cdot 2 \cdot 2 \pi}+1}$ \\
\hline
\end{tabular}

A performance comparison between the model-based and the VRFT calibration approaches is shown in Fig. 10, where two important results can be highlighted. Firstly, the perfect matching for both tuning of the target closed-loop model and secondly the high similarity between the responses of the two calibrated controllers. A quantitative comparison can be also done by inspecting the resulting VRFT control parameter are in Table IV against the model-based ones in Table II.

TABLE IV

DATA-DRIVEN CONTROL PARAMETERS

\begin{tabular}{l|c|c|c|c}
\hline & \multicolumn{2}{|c|}{ Position Control } & \multicolumn{2}{c}{ Pressure Control } \\
\hline proportional gain & $\frac{A}{m m}$ & 16.1133 & $\frac{m m}{b a r}$ & 0.0067 \\
\hline integral gain & $\frac{A}{m m \cdot s}$ & 484.5347 & $\frac{m m}{b a r \cdot s}$ & 0.8335 \\
\hline derivative gain & $\frac{A \cdot s}{m m}$ & 0.1002 & - & - \\
\hline
\end{tabular}




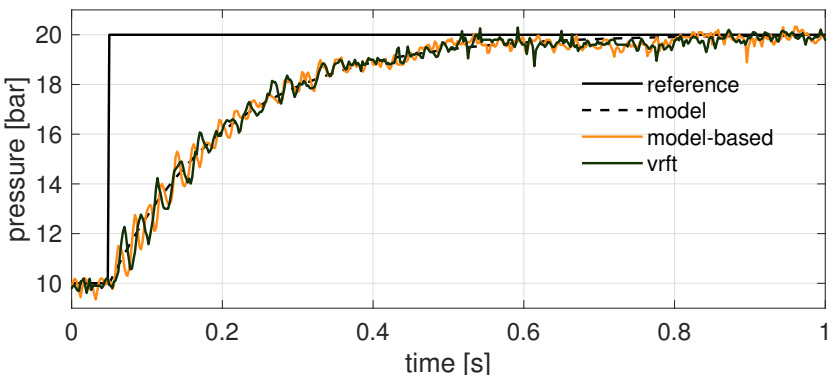

Fig. 10. Step response of the model-based and data-driven pressure controlled systems compared with the desired response.

\section{Recalibration during real braking profile tracking}

The last point considered to improve the flexibility of the controller is the application of the previous algorithm on data coming from real braking profile tracking (see Fig. 11 ), in order to achieve a recalibration of the control system without the need of an ad-hoc experiment, like the openloop PRBS previously described. The traditional framework

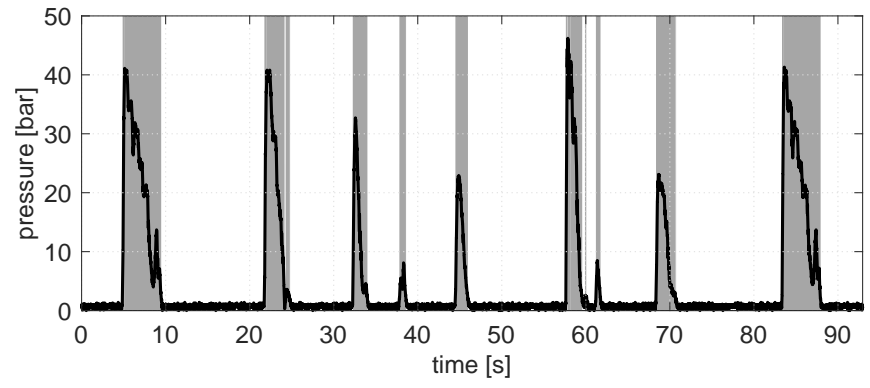

Fig. 11. Real pressure profiles, where the data used for VRFT are highlighted.

of VRFT is those of a batch algorithm. However, equation (8) can be written in its recursive form. Fig. 11 shows a series of real braking used as data-set to test the recursive formulation; notice that only valid data are used, i.e when a minimum braking pressure is applied. Fig. 12 shows the evolution of the controller parameters, starting from a wrong guess. All parameters are normalized with respect to the values in Table IV, so their convergence towards one means that a proper controller tuning has been reached. Notice that, given their smaller impact on the closed-loop behaviour, the convergence of the derivative gain of the position controller and the proportional gain of the pressure one requires more time.

\section{CONCLUSIONS}

In this work, a data-driven procedure for the calibration of a Brake-by-Wire actuator has been addressed and compared to a standard model-based one. The procedure is based on the VRFT extension for cascaded control systems and experimentally proves to yield equivalent results w.r.t. the model-based approach, with the need of lighter experimental campaign. Additionally, it is shown how the method can be directly applicable during the normal operation of the
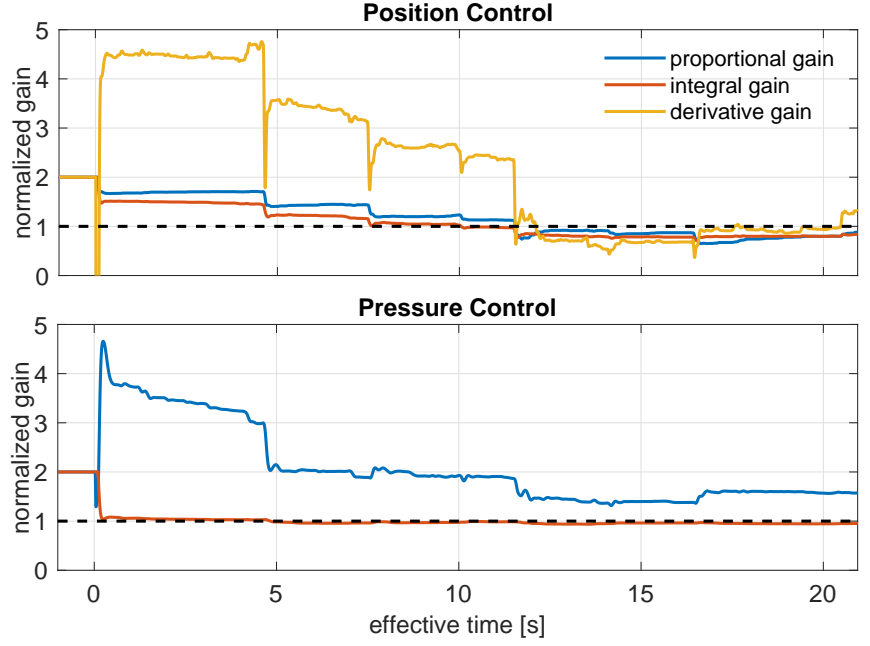

Fig. 12. VRFT gains resulting from real-time data normalized with respect to the offline VRFT gains, staring from a wrong initial condition (the double of the values in Table IV).

actuator without the need for ad-hoc experiments, opening the way to an automatic maintenance of the BBW closedloop performances.

\section{REFERENCES}

[1] F. Todeschini, M. Corno, G. Panzani, S. Fiorenti, and S. M. Savaresi, "Adaptive cascade control of a brake-by-wire actuator for sport motorcycles," IEEE/ASME Transactions on Mechatronics, vol. 20, no. 3, pp. 1310-1319, 2015.

[2] K. Chau, "21 - Pure electric vehicles," in Alternative Fuels and Advanced Vehicle Technologies for Improved Environmental Performance, R. Folkson, Ed. Woodhead Publishing, 2014, pp. 655-684.

[3] A. Dardanelli, G. Alli, and S. M. Savaresi, "Modeling and control of an electro-mechanical brake-by-wire actuator for a sport motorbike," IFAC Proceedings Volumes, vol. 43, no. 18, pp. 524-531, 2010, 5th IFAC Symposium on Mechatronic Systems.

[4] F. Todeschini, M. Corno, G. Panzani, and S. M. Savaresi, "Adaptive position-pressure control of a brake by wire actuator for sport motorcycles," European Journal of Control, vol. 20, no. 2, pp. 79 - 86, 2014.

[5] R. de Castro, F. Todeschini, R. E. Araújo, S. M. Savaresi, M. Corno, and D. Freitas, "Adaptive-robust friction compensation in a hybrid brake-by-wire actuator," Proceedings of the Institution of Mechanical Engineers, Part I: Journal of Systems and Control Engineering, vol. 228 , no. 10 , pp. $769-786,2014$

[6] F. Todeschini, S. Formentin, G. Panzani, M. Corno, S. M. Savaresi, and L. Zaccarian, "Nonlinear pressure control for bbw systems via deadzone and antiwindup compensation," IEEE Transactions on Control Systems Technology, vol. 24, no. 4, pp. 1419-1431, 2016.

[7] M. C. Campi, A. Lecchini, and S. M. Savaresi, "Virtual reference feedback tuning: a direct method for the design of feedback controllers," Automatica, vol. 38, no. 8, pp. 1337-1346, 2002.

[8] S. Formentin, A. Cologni, D. Belloli, F. Previdi, and S. M. Savaresi, "Fast tuning of cascade control systems," IFAC Proceedings Volumes, vol. 44, no. 1, pp. 10243-10248, 2011, 18th IFAC World Congress.

[9] P. Panizza, D. Invernizzi, F. Riccardi, S. Formentin, and M. Lovera, "Data-driven attitude control law design for a variable-pitch quadrotor," in 2016 American Control Conference (ACC), 2016, pp. 4434 4439.

[10] K. J. Astrom and R. M. Murray, Feedback Systems: An Introduction for Scientists and Engineers. USA: Princeton University Press, 2008.

[11] L. Ljung, "Recursive least-squares and accelerated convergence in stochastic approximation schemes," International Journal of Adaptive Control and Signal Processing, vol. 15, no. 2, pp. 169-178, 2001. 